Dermatologische Zeitschrift. 1930;58:I-IV

\title{
Contents, Vol. 58, 1930
}

Inhaltsverzeichnis.

Originalarbeiten.

Boas, Ha raid, und T. Sodemann, Der Einfluß der kongenitalen Syphilis auf Schwangerschaft, Geburt, Wochenbett und Nachkommen-

schaft 1

Brill, E., Syphilimetrie nach Vernes mit Hilfe des Zeißscheo Stufen-photometers. (Ein kritischer

Vergleich mit den Ergebnissen der

Wassermannschen Reaktion) 386

Dohi, Sh., Über Trichotillomanie 236

Geiger, Richard, Über Schweißdrüsennävi 240

Gelder, R. J. van, Bemerkungen zu der Mitteilung von Dr. Wo hi stein:

Infektion der Haut beim Menschen 368

Gottron, H., Beitrag zur Ätiologie der Melkerknoten207

Heller, Julius, Zur Kasuistik seltenerNagelerkrankungen. XXI. Syphi-

lonychia congenita ulcerosa ungium sanata bei einem 20 jährigen Mann 301

- $\quad$ XXII. Heilung eiøer schweren trophischen Nagelerkrankung trotz

Fortbestehen schwerster trophischer Hautsymptome 303

Jordan, Arthur, Das Lupus-vulgaris-Karzinom 136

Langer, Erich, Die Gonorrhoe der paraui ethralen Gänge bei der Frau,

ein Fall seltener Lokalisation derselben 158

Löhe, H., und D. Nitschke, Ungewöhnlicher Sitz multipler Spontan-

frakturen bei Tabes dorsalis 150

Mallinckrodt-Haupt,Asta von, Milbenerkrankungen beim Menschen

(Schl·uß). Ein neuer Trombidioseherd in der Eifel 24

March · ionini , Alfred, Zur Pathogenese und Differentialdiagnose

dyshidrotischer und dyshidrosiformer Bläschenerkrankungen derHände

und Füße 222

- $\quad$ und Berta Ottenstein, Die Goldsolreaktion des Liquor cerebro-

spinalis mit elektropositivem Hydrosol

Meyer-Buley, Hans, Die Frühdiagnose von noch im Kiefer ver-

borgenen Hutchinson-Zähnen mit Hilfe des Röntgenbildes 313

Nitschke, D., siehe Löhe, H.

Ottenstein, Berta, siehe Marchionini, Alfred.

Rille, J. H., Die Hauterscheinungen der Pellagra

Sellei, Josef, Formen und Ursachen der als Chloasma uterinum benannten Hyperpigmentation des Gesichtes 17

Sodemann, T., siehe Boas, Ha raid.

Stümpke, G., Zur Frage der Thalliumvergiftung $\quad 10$

Wohlstein, Emanuel, siehe Gelder, R. J. von. 
Zoon, J. J., Blastomyocosis cutis durch Monilia Fioccoi mit positiver

Blutkultur $\quad 356$

Edmund Saalfeld $\uparrow \quad 124$

IV

Inhaltsverzeichnis.

Gesellschaftsberichte. seite

Berliner Dermatologische Gesellschaft. Sitzung vom 12. XI. 1929

Sitzung vom 29. XL 1929

163

Sitzung vom 10. XII. $1929 \quad 248$

Sitzungen vom 10. I. und 11.11. 1930

371

Kölner Dermatologische Gesellschaft. Sitzungen vom 25. X. u. 29. XI. 1929

- $\quad$ Sitzung vom 31.1.1930 420

Herbsttagung der Vereinigung rheinisch-wcstfäliseher Dermatologen in

Mü口ster i. W. am 26. u. 27. X. $1929 \quad 400$

Gemeinsame Tagung der Niederländischen Dermatologen-Vereinigung und der Vereinigung rheinisch- $\Lambda$ vestfäliseher Dermatologen in Köln

am 25. und 26. V. 1929 63

Royal Society of Medicine, Section of Dermatology. Sitzungen vom

16. V. und 20. VI. $1929 \quad \ldots .76$

- $\quad$ Tagung vom 17. X. $1929 \quad 264$

North of England Dermatological Society. (Nordenglische Dermatologische Gesellschaft.) Sitzung vom 10. V. 1929 in Liverpool 80

- $\quad$ Sitzung vom 11. X. $1929 \quad 266$

Société française de Dermatologie et de Syphiligraphie. Sitzung vom

14. II. 1929 177

Gesellschaft für Haut- und Geschlechtskrankheiten in Kiew. Sitzungen

vom 17. II. und 7. X. 1929

The Victorian Association of Dermatology. Sitzung vom 12. VI. 1929 in

Melbourne 80

Periodische Literatur. Zusammengestellt von Dr. Arthur Alexander-

Charlottenburg $\quad 85,185,267,421$

Buchbesprechungen 121, 202, 296, 439

Zum 25. Jahrestag der Eлtdeckung der Syphilis-Spirochaeta. Von W. Frieboes-Rostock 299

Personalien und Tagesnachrichten 124, 206, 300, 440

Zum 70. Geburístag von Prof. Dr. Fabry 439

Druckfehler-Berichtígung 126

Sachregisíer 442

Nam en register $\quad 451$ 